Instituto Internacional de Investigación y Desarrollo Tecnológico Educativo INDTEC, C.A.

DOI: https://doi.org/10.29394/Scientific.issn.2542-2987.2019.4.12.2.47-67

OAI-PMH: http://www.indteca.com/ojs/index.php/Revista Scientific/oai

Artículo Original / Original Article

\title{
Estrategias Ambientales en la Formación Ecoturística de los Estudiantes de Educación Primaria
}

Autora: Belkis Esteban Moncada Universidad Pedagógica Experimental Libertador, UPEL belkisesteban2016@gmail.com

Barinas, Venezuela https://orcid.org/0000-0002-1012-781X

Resumen

La presente investigación presentó como objetivo general proponer estrategias ambientales en la formación ecoturística de los estudiantes de Educación Primaria, dirigido a los docentes de la Escuela Básica Sebastián Araujo, municipio Pedraza, estado Barinas. El estudio centrado en la naturaleza cuantitativa, utilizando el proyecto factible apoyado en un diseño de campo. Se tomó como población veintiún (21) docentes de la institución antes mencionada. Asimismo, se manejó la técnica de la encuesta y como instrumento un cuestionario constituido por dieciséis (16) ítems con escalamiento tipo Likert. Para la validez se utilizó el juicio de expertos, empleando la confiabilidad de Coeficiente ( $\alpha$ ) Alfa de Cronbach, los cálculos arrojaron como valor 0,86 considerada muy alta la confiabilidad. De acuerdo a las conclusiones se diseñaron las estrategias ambientales atendiendo a las dimensiones: (a) Conocimiento Ambiental, permite conocer la importancia del ambiente en su componente natural vinculado al entorno; (b) Vinculación Socio Turismo Ambiental, permite atender el espacio geográfico y el tiempo histórico significativo, interactuando la familia, escuela y comunidad en los escenarios de convivencia; (c) Formación Turístico Ambiental, Capacitación de Guías Turístico Ambientales, accediendo a desarrollar la generación de actitudes a partir de espacios que concedan pensamientos y reflexión en función a los procesos ambientales, sociales, económicos, políticos y educativos y; (d) Afiliación del Legado Cultural en el axioma de habilidades y planes de referencia a la cultura local.

Palabras clave: ambiente; educación; ecoturismo.

Fecha de Recepción: 12-11-2018

Fecha de Aceptación: 09-02-2019 


\title{
Environmental Strategies in the Ecotourism Training of Primary Education Students
}

\begin{abstract}
The present investigation presented as a general objective to propose environmental strategies in the ecotourism formation of the students of Primary Education, directed to the teachers of the Basic School Sebastián Araujo, municipality Pedraza, state Barinas. The study focused on the quantitative nature, using the feasible project supported by a field design. Twenty-one (21) teachers from the aforementioned institution were taken as a population. Likewise, the survey technique was handled and as a tool a questionnaire consisting of sixteen (16) items with Likert-type scaling. For the validity, expert judgment was used, using the reliability of Coefficient ( $\alpha$ ) Alpha of Cronbach, the calculations gave as value 0.86 considered reliability very high. According to the conclusions, the environmental strategies were designed according to the dimensions: (a) Environmental Knowledge, allows to know the importance of the environment in its natural component linked to the environment; (b) Socio-Environmental Tourism Linking, allows serving the geographic space and significant historical time, interacting with the family, school and community in the scenarios of coexistence; (c) Environmental Tourism Training, Training of Environmental Tourist Guides, acceding to develop the generation of attitudes from spaces that grant thoughts and reflection according to environmental, social, economic, political and educational processes and; (d) Affiliation of the Cultural Heritage in the axiom of skills and plans of reference to the local culture.
\end{abstract}

Keywords: environment; education; ecotourism.

Date Received: $12-11-2018$

Date Acceptance: 09-02-2019 


\section{Introducción}

En los últimos años, el mundo ha comenzado a evolucionar de forma apresurada, por lo tanto, las naciones se han interesado en prepararse para aprovechar el potencial del pensamiento humano en el desarrollo tecnológico, económico, político y social; sembrando una instrucción y una cultura en función de fortalecer la creatividad, la disciplina y el trabajo. En efecto, existe una elevada evidencia que la educación es el medio esencial para afrontar los nuevos desafíos y alcanzar una excelente producción y colocación de los riquezas y productos que demanda la sociedad. Al respecto, Santander (2010a), expone que:

La educación debe comprenderse, en estos tiempos, como aquella expresión concisa de identidad nacional y conocimiento, erigiendo un ser social que resignifique su localidad y logre obtener de ella los aspectos indispensables para generar una visión universal que lo proyecte como sociedad mundial (pág. 66).

Por consiguiente, la finalidad de la educación es instruir a niños, jóvenes y adultos en tes proactivos, sinérgicos, analíticos y autónomos; generando ser más interactivos, protagonistas en todas las acciones inherentes a la triada familia-escuela-comunidad; fortalecimiento los valores de amor, cooperación y respecto hacia el país, sus colaboradores y el ambiente, además, deben conocer los saberes propios de su región y zona cultural, para sentirse orgullosos de ellos. Desde este marco referencial, se plantea el papel de la educación ambiental, Gabaldón (2006), citado por Requena (2018): refiere que: "la identificación de los problemas ambientales y cómo controlarlos requiere un nuevo tipo de educación que a través de un juicio teórico y práctico del medio biótico y físico cree el interés y el amor por la naturaleza" (pág. 296).

De esta manera, dentro de los objetivos del quehacer educativo esencialmente se plantea en la instrucción el fomentar un conocimiento ambiental sustentado en principios, que permitan consolidar el conjunto de 
habilidades para el reconocimiento de los diferentes escenarios de actuación ambiental, no solamente en la academia, sino también en todo lo relacionado con los aspectos comunitarios, principalmente aquellos que vienen desde el hogar, en función de fomentar la sostenibilidad en la vida de los ciudadanos.

Por consiguiente, en Venezuela, específicamente en el subsistema de Educación Primaria, los retos actuales se concentran en enaltecer la calidad educativa en aquellos espacios que han sido trabajados e incluir al currículo y a las acciones diarias: el aprendizaje continuo y efectivo de los estudiantes en el aspecto geográfico y social de la comunidad en la cual se encuentra la escuela. No obstante, Rengifo, Quitiaquez y Mora (2012):

Los procesos educativos que incluyen la enseñanza de la educación ambiental pretenden tomar conciencia del individuo y la sociedad de igual forma la capacitación conforman un proceso que permite que los seres humanos y las sociedades desarrollen plenamente la capacidad de conocimiento del mundo y la realidad interpretarlos, explicarlos y vivir sus circunstancias. La educación ambiental promueve el desarrollo y aumenta las posibilidades de la población para emprender su desenvolvimiento (pág. 4).

En efecto, la educación ambiental se crea como estrategia dentro del tejido de la educación, como indeleble en el sujeto, razón por la cual la escuela y comunidad son, al mismo tiempo, objeto y sujeto en el desarrollo de acciones ambientales, propiciando el sentido de pertenencia a la situación social y educativa; profundizando en la participación de los actores sociales, con la finalidad de alcanzar la construcción de un sistema educativo que abarque el proceso integral de los individuos, de acuerdo a sus necesidades, intereses y expectativas institucionales y comunitarias. En este contexto, toma vigencia el llamado ecoturismo o turismo ecológico referido por Villegas (2012), como:

Nueva tendencia del turismo alternativo el cual enfoca las actividades turísticas privilegiando la sustentabilidad, la preservación, la apreciación del medio, tanto natural como cultural, acogiendo y sensibilizando a los viajantes desde el 
valor ético del contexto y, que supone como fundamental el bienestar de las poblaciones locales (pág. 21).

El autor refiere, el ecoturismo es una de las expresiones más evidentes en la actualidad, de la mayor conciencia e interés de planificar y trasportar las acciones turísticas de forma responsable, beneficiando la sustentabilidad y el potenciar la economía a niveles regional y nacional. Al considerar, dentro del contexto educativo la formación en ecoturismo, considerada como una estrategia para formar en valores ambientales significativos para el individuo, tales como: respeto, cooperación, tolerancia, comportamiento responsable con el ambiente, entre otros.

En relación a ello, Martín (2010a), refiere que: "el ecoturismo educativo no es una actividad económica fría, sino que se nutre de las experiencias, por ello requiere una mayor formación humanista desde enfoques multidisciplinarios. El ecoturismo es un baluarte de la educación" (pág. 3); es decir, el fundamento educativo del ecoturismo radica en propiciar redes de interpretación, en función de no informar gran cantidad de datos, sino que se describan contenidos y significados que posibiliten la elaboración de planes efectivos, con una visión integrada y regida en valores de: amor, equidad, cooperación, respeto, entre otros.

Ciertamente, tal como lo expresa Martín (2010b): "la planificación educativa del docente en el aula, esporádicamente aborda la temática ambiental, por lo que la formación educativa en materia de ecoturismo no es un área desarrollada frecuentemente en el contexto educativo" (pág. 13); en otras palabras, al referirse a nivel local, especificamente, en las instituciones educativas del estado Barinas, acerca del desarrollo de estrategias ambientales, por parte de los docentes, para orientar una formación ecoturística en los estudiantes, lo cual incidirá positivamente en el proceso educativo integral. 
En este sentido, se destaca que tal situación no es ajena a la realidad que presenta la Escuela Sebastián Araujo, ubicada en el municipio Pedraza, dado que a través de la observación directa se percibe, el desconocimiento con respecto al diseño y desarrollo de estrategias ambientales, por parte de los docentes, para abordar la educación ambiental desde un contexto que permita afianzar valores en los niños en cuanto la identidad regional y local, los bienes materiales y espirituales que se deben cuidar y proteger en común, para rescatar y conservar los haberes patrimoniales e idiosincrasia de su comunidad. De allí, nace la motivación de efectuar una investigación que tiene como objetivo general proponer estrategias ambientales en la formación ecoturística en los estudiantes de Educación Primaria, dirigida a los docentes de la Escuela Básica Sebastián Araujo, municipio Pedraza, estado Barinas.

La situación planteada motivó el abordaje del presente estudio, estableciéndose un esquema de interrogantes, a saber: ¿Qué conocimientos tienen los docentes de la Escuela Básica "Sebastián Araujo", municipio Pedraza, estado Barinas acerca de las estrategias ambientales y la formación ecoturística?; ¿Es factible educativamente, económicamente e institucionalmente la ejecución de estrategias ambientales para la formación ecoturística de los integrantes de la Escuela Básica Sebastián Araujo?; y ¿Cómo será el diseño de estrategias ambientales en la formación ecoturística de los docentes de la Escuela Básica Sebastián Araujo, municipio Pedraza, estado Barinas?

\section{Antecedentes, Teorías y Conceptos}

\subsection{Antecedentes de la Investigación}

Las referencias del estudio representan la revisión de trabajos ya realizados, trabajos de ascensos e informes científicos que tienen correspondencia con la investigación objeto de estudio. Es así como, Palella y Martins (2012): exponen que los antecedentes son los "diferentes trabajos 
realizados por otros estudiosos sobre el mismo problema" (pág. 63). Por consiguiente, los antecedentes de la investigación permiten la revisión de aspectos que involucran estudios anteriores a éste, para darle apoyo, tomando en consideración el autor, año de la investigación, propósito, procesos metodológicos, resultados, conclusiones y aportes relevantes para con ésta. Seguidamente se presentan algunos de ellos.

En este sentido, Molano (2013a), planteó como objetivo: "caracterizar las concepciones y prácticas docentes sobre educación ambiental que se promueven en los programas de educación de las universidades bogotanas y establecer sus implicaciones en la construcción de currículos para dichos programas" (pág. 201).

Por lo tanto, el abordaje metodológico estuvo centrado en un estudio de caso de tipo instrumental. Asimismo, Molano (2013b), indica que: "los programas de educación de las universidades de Bogotá, para indagar en las concepciones de los/las docentes sobre educación ambiental y caracterizar sus prácticas pedagógicas de acuerdo con dichas concepciones" (pág. 202). Por consiguiente, definidas las ideas y experiencias, se instituyeron las discrepancias en los currículos presentes de las jurisdicciones de educación y se esbozan acciones-directivas del plan de estudio, en la cual se enmarca en la capacitación ambiental en los estudiantes que se preparan las carreras de educación en las instituciones de Bogotá que se ofrecen. Igualmente, Molano (2013c), señala:

El universo está constituido por veintinueve (29) universidades en Bogotá, la población fue quince (15) universidades que ofrecen setenta y nueve (79) programas de licenciatura en educación con diferentes énfasis y la muestra la constituyó nueve (09) universidades que ofrecen veintiún (21) programas de licenciatura en educación, en la cual se dan cátedras y asignaturas específicas sobre educación ambiental (pág. 214).

La investigación antes expuesta, aporta elementos teóricos relacionado 
con la educación ambiental, dado que se busca cambios de actitud, postura crítica, reflexiva de la conservación, resguardo, protección, transformación, desarrollo sostenible y sustentable, resolución de problemas relacionado con el ambiente.

En este orden y dirección, Zuccarro (2010), se propuso como objetivo de estudio: "elaborar una propuesta educativa ambiental centrada en el turismo sostenible dirigida a la población que utiliza los ecosistemas dulce acuáticos: Paso La Balsa y Río La Acequia del Estado Barinas" (pág. 129). El paradigma asumido, fue la investigación cualitativa, razón por la cual busca la descripción, indagación, se declaran los fenómenos sociales. La técnica empleada en la recolección de la información fue la encuesta, utilizando dos cuestionarios de opinión aplicados al universo seleccionado.

El antecedente contribuye con elementos teóricos relacionado con la sensibilización ambiental en los individuos hacia la valoración, convivencia y experiencia que se tiene con el ambiente y el contexto en cual se desenvuelve. La educación ambiental conduce a la formación holística del ser humano en cuanto a lo educativo, social, económico, político y cultural; entramando acciones hacia la consolidación del turismo.

\subsection{Estrategias Ambientales}

La terminología de estrategias ambientales, según Sánchez (2012a): "fue usada inicialmente en la Agenda 21" (pág. 21). El mismo consistió en un pliego inscrito en la Cumbre de la Tierra, celebrado en el año 1992 en Brasil, en la cual se impulsa a los gobiernos patrocinen acciones nacionales en el desarrollo sostenible y sean elaboradas con la extensa participación de los sectores. De acuerdo con Romero (2010a), las estrategias ambientales corresponden al:

Producto de un conjunto de acciones lógicas y creativas que conducen a la formulación de objetivos amplios, de políticas 
principales y de asignación de recursos para lograr las metas trascendentales, en la búsqueda de una mejor posición y una respuesta más coherente ante el entorno actual y futuro, involucrando a la sociedad como ente de acción y de razón (pág. 98).

Por consiguiente, una estrategia ambiental efectiva se da por medio de auspiciar información actualiza del tema y con una efectiva comunicación, colocando la transcendencia de los individuos a recursos prácticos que puedan ser utilizados en la vida diaria; brindando la posibilidad de acceso a una mayor indagación, seguimiento y control a dichas estrategias y la inscripción de los medios de comunicación y sistema educativo.

A través de las estrategias ambientales se puede evidenciar la representación estratégica de la educación ambiental en función de fomentar e implementar el desarrollo sostenible. No obstante, es indiscutible que las acciones educativas, por sí mismas, es insuficiente responder al desafío ambiental; por lo que, para ayudar con efectividad a optimizar el ambiente, las actividades educativas deben enlazarse con las leyes, políticas, normas de intervención y la toma de decisiones de los entes de gobierno en correspondencia con el ambiente.

\subsection{Ambiente}

La concepción de ambiente es comúnmente usado con dos vínculos diferentes: una estancada y restrictiva, que reseña al contexto, material, tangible, mesurable y, que en momentos resulta preciso, en relación al sujeto; y otro, complejo, dinámico, amplio y comprensivo, observándose simplemente, por sus expresiones y efectos en la calidad de la vida, que envuelve al individuo y lo hace parte sólida en la interacción sociedad-ambiente. De allí,

percibe al ambiente como algo majestuoso y complejo. Del mismo modo, Martínez (2010), expone que: 
La educación ambiental consiste en lograr que los individuos y las colectividades comprendan la naturaleza compleja del ambiente natural y del creado por el ser humano, que sea resultado de la interacción de sus aspectos biológicos, físicos, sociales, económicos y culturales; y adquieran los conocimientos, los valores, los comportamientos y las habilidades prácticas (pág. 101).

El autor refleja, que la educación ambiental representa un proceso dinámico, emprendedor e interactuante entre elementos naturales, sociales, biológicos, culturales, políticos, educativos y psíquicos. A su vez se encuentra inmerso en un sistema holístico, es decir, que se interconectan de forma continua; incidiendo en transformaciones antepuestas ya existentes en un contexto predeterminado.

\subsection{Educación Ambiental}

La educación ambiental trata aspectos globales de manera crítica, sus efectos e interrelaciones en una visión holista del tejido social, formativo y auténtico, entre ellos: ciudad, armonía, derechos humanos, libertad, vigor, hambre, disminución de la vegetación y animales, marginalidad, entre otros. Al respecto, Sánchez (2012b), indica que:

Se concibe como un proceso permanente en el que los individuos y la colectividad cobran conciencia de su medio y adquieren los conocimientos, los valores, las competencias, la experiencia y la voluntad capaces de hacerlos actuar individual y colectivamente para resolver los problemas actuales y futuros del medio ambiente (pág. 49).

Desde esta perspectiva queda claro que se trata de un proceso que afecta a las personas, que tiene una tendencia hacia la actitud y el comportamiento, sin embargo, no debe pensarse solamente en la adquisición de un conjunto de conocimientos y capacidades, sino en la formación integral del individuo; accediendo a educar para la vida, enfrentando adecuadamente 
las dificultades de la correspondencia ser-ambiente-sociedad, ampliando su conceptualización y temática, llevado a ponderar el papel de la cultura de los pueblos, la revitalización de lo indígena, el vital papel de la mujer, la inserción de los elementos físicos, naturales, económicos, políticos, técnicos, históricos, morales y éticos.

Se insta en la necesidad del compromiso particular y colectivo, en el conocimiento transformador y en la perspectiva interdisciplinaria que define a la educación ambiental; incidiendo en la razón ética que debe producir, quizás como idea clara y relevante. Se concreta como una educación que no es imparcial sino ideal, es un suceso gubernamental, asentado en principios para la innovación general. De igual manera, trata aspectos totales de manera crítica, sus raíces e interrelaciones con una visión holística del entorno social, científico, cultural, histórico y educativo; profundizando en la resignificación del individuo como ser, entre otros aspectos relevantes en la formación de los ciudadanos.

\subsection{Ecoturismo}

El ecoturismo tuvo sus orígenes hace varias décadas y, desde sus inicios ha ido avanzando su popularidad, intrínsecamente, en los espacios de la conservación y la actividad turística. El ecoturismo, llamado también turismo alternativo, nace como una oposición al supuesto de masas, esta busca la integración del desarrollo sustentable y el turismo. Al respecto, Santander (2010b), lo define como:

La particularidad turística de buscar la responsabilidad de acercar a los individuos en viajar o visitar lugares naturales, con el propósito de generar recreación, esparcimiento y disfrute en valorar los elementos que se encuentran en la naturaleza; concienciando y profundizando en la conservaciónpreservación de la misma (pág. 5).

En otras palabras, el ecoturismo se evidencia cuando se desarrollan 
actividades de concienciación, capacitación y se promocionan acciones de conservación que enseñen al entendimiento del entorno ambiental, logrando la transferencia hacia los visitantes, para que éstos adopten un enfoque de transformación y reflexión, lo que hace apreciar que se está instruyendo, que se está contribuyendo a los cambios de actitud en niños, jóvenes y adultos; incidiendo en ellos en cuanto a la conservación y conservación del espacio. La actividad ecoturística contribuye de manera significativa a la educación ambiental y a la concientización ecológica. Para lograr esto, es importante desarrollar acciones turísticas con fines educativos que optimicen la experiencia de las personas y, aumente el valor de los espacios naturales.

\subsection{Formación}

La educación, es un proceso por medio de la cual se enfatiza en el fortalecimiento de las competencias y habilidades del individuo, con la finalidad que se incorpore íntegramente a la sociedad; perfilándose en la indagación del del bienestar del colectivo. Por su parte, Fuentes (2013), expone: "la formación se concibe como un proceso social y cultural que obedece al carácter de la integridad del desarrollo de la capacidad transformadora humana que se da en la dinámica de las relaciones entre los sujetos en la sociedad" (pág. 45); sígnica que la formación accede en el individuo, para profundizar en la temática social, ambiental y cultural; revelándose contradictoriamente en los procesos del desarrollo humano; de manera sistémica, individualmente y colectivamente en los sujetos y en sus conocimientos.

Consecuentemente, la formación docente representa una inherencia en la praxis y la teoría diaria de los maestros que mantienen funciones como profesionales en el ámbito educativo; los mismos son los encargados de iniciar la autoformación como aspecto principal de potenciar, generar cambios y transformaciones internamente en los individuos y el entorno en la cual cultivan su profesión. Sin embargo, es importante acotar que la formación permanente 
no requiere de la exigencia de un programa determinado, es decir, requiere de la motivación interna o personales que tiene cada maestra.

\section{Metodología}

El estudio fue focalizado en el paradigma cuantitativo, razón por la cual se orienta bajo la búsqueda de resultados confiables empleando métodos y técnicas pertinentes para ello. También se encuentra inserto en la modalidad de proyecto factible apoyado en un estudio de campo. Al respecto, en el Manual de Trabajos de Grado de Especialización y Maestría y Tesis Doctorales de la Universidad Pedagógica Experimental Libertador (2016), se refleja que:

El Proyecto Factible consiste en la elaboración y desarrollo de una propuesta de un modelo operativo viable para solucionar problemas, requerimientos o necesidades de organizaciones 0 grupos sociales puede referirse a la formulación de políticas, programas, tecnologías, métodos o procesos. E proyecto debe tener apoyo en una investigación de tipo documental, de campo o de un diseño que incluya ambas modalidades (pág. 21).

Interpretando la cita, el proyecto factible se enmarca en la elaboración de proposiciones viables que busquen mejorar alguna situación o problema en una comunidad, instituciones, organizaciones y sociales. Es por ello, que en el presente estudio pretender proponer estrategias ambientales en la formación ecoturística a los estudiantes de Educación Primaria, en la cual sean promovidas por los docentes de dicho nivel.

Por otra parte, la población y muestra estuvo constituida por veintiún (21) docentes que laboran en la Escuela Básica "Sebastián Araujo", ubicada en el municipio Pedraza del estado Barinas. De allí que, la técnica usada fue la observación y como instrumento un cuestionario con escala Likert: Siempre, Casi Siempre, Algunas Veces, Casi Nunca y Nunca. El análisis de los datos se efectuó con la tabulación, análisis y descripción los datos recolectados. 


\section{Resultados}

En este apartado denominado resultados, se representa las distintas operaciones que se siguieron para procesar e interpretar los datos obtenidos de las respuestas emitidas por los sujetos que conformaron la muestra, en este caso, los docentes de la Escuela Básica "Sebastián Araujo". La misma se ejecutó de manera porcentual, lo cual permitió explicitar el comportamiento de los resultados en gráficos de barras. A continuación, se presentan:

Gráfico 1. Distribución de la frecuencia de la variable: estrategia ambiental en su dimensión: pedagógica e indicadores características y promoción.

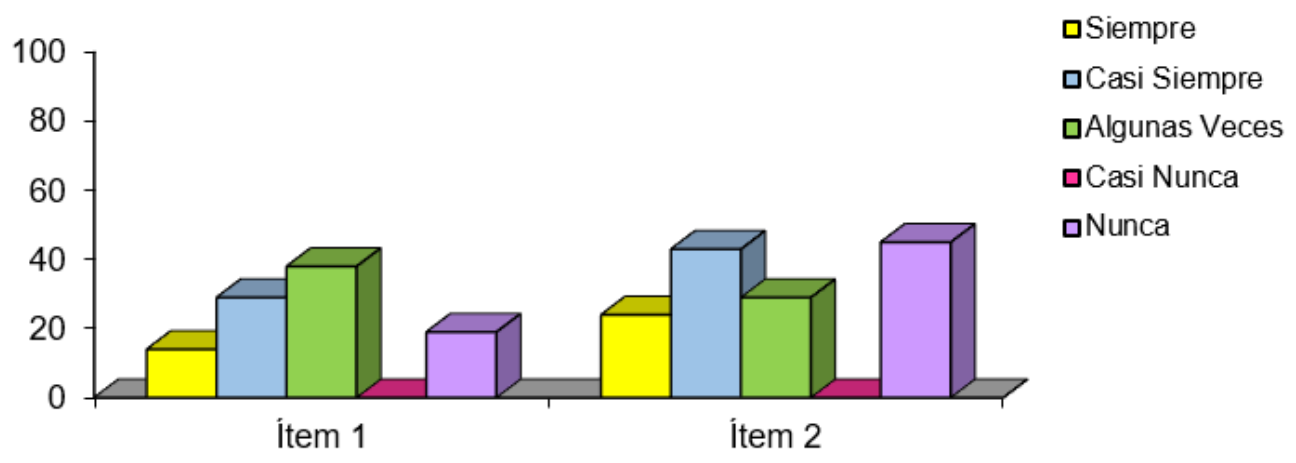

Fuente: La Autora (2018).

Los datos obtenidos en el gráfico 1, correspondiente al ítem 1, el $38 \%$ de los encuestados expresaron que "Algunas Veces" los docentes manejan el conocimiento que las características didácticas de las estrategias ambientales conducen al conocimiento ambiental el 29\% "Casi Siempre", el 14\% "Siempre" y el 19\% "Nunca. Para el ítem 2, el 43\% indicaron que "Casi Siempre" a partir del desarrollo de estrategias ambientales se estimulan en los estudiantes la promoción de responsabilidad hacia el ambiente, el 29 \% "Algunas Veces", el $24 \%$ "Siempre" y el $4 \%$ "Nunca".

Los resultados obtenidos conducen a inferir que los profesionales de la docencia expusieron que con poca frecuencia manejan el conocimiento que las características didácticas de las estrategias ambientales conducen al 
conocimiento ambiental. Sin embargo, la gran mayoría de los profesores consideran que partir del desarrollo de estrategias ambientales, se potencia en los estudiantes la promoción responsabilidad hacia el ambiente.

En efecto, las estrategias ambientales, según Romero (2010b), plantea que: "adquieren un sentido pedagógico y de sensibilización para el proceso educativo, cuando contribuyen a que el aprendizaje se genere de forma natural en la construcción del pensamiento" (pág. 99); profundizando en las competencias, habilidades, capacidades de pensamiento individual y colectivo.

Gráfico 2: Distribución de la frecuencia de la variable: formación ecoturística en su dimensión: educativa e indicadores conocimiento e importancia.

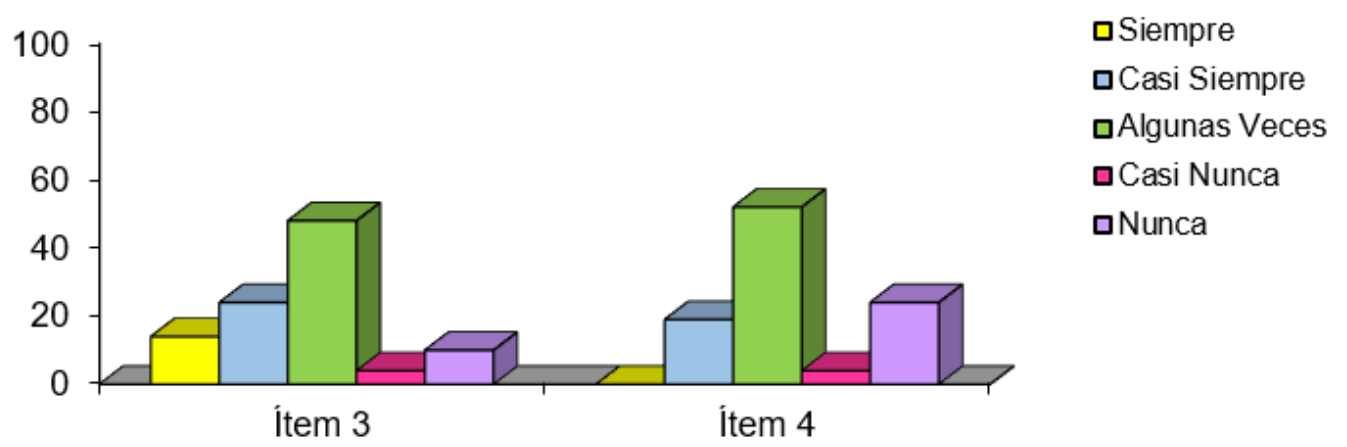

Fuente: La Autora (2018).

En el gráfico 2, en el ítem 3 el $48 \%$ de los docentes respondieron "Algunas Veces" manejan el conocimiento que el ecoturismo consiste en desarrollar actividades de formación, sensibilización y promoción de acciones en la conservación del ambiente, el 24\% "Casi Siempre", el 14\% "Siempre", el 10\% "Nunca" y el 4\% "Casi Nunca". El ítem 4, el 52\% manifestaron "Algunas Veces" consideran que la aplicación de estrategias ambientales es importante en la formación ecoturística de los estudiantes, el 24\% "Nunca", el 19\% "Casi Siempre" y el 4\% "Casi Nunca".

Los datos analizados conducen a interpretar que los docentes 
medianamente manejan el conocimiento que el ecoturismo consiste en desarrollar actividades de formación, sensibilización y promoción de acciones en la conservación del ambiente; así como también consideran que la aplicación de estrategias ambientales es relevante en la formación ecoturística de los educandos.

En orden y dirección, Santander (2010c), señala que: "el conocimiento en acciones ecoturísticas permite consolidar y reforzar el abordaje de diferentes áreas del conocimiento" (pág. 67); que sustenten además el enfoque educativo participativo de la escuela hacia el entorno; involucrando a cada uno de los integrantes de la comunidad hacia actividades referentes a la conservación y preservación del ambiente.

\section{Conclusiones y Recomendaciones}

En la aplicación del instrumento a los docentes se evidenciaron: Desconocimiento acerca del tipo de estrategias ambientales que desde la acción educativa respondan al desafío ambiental y contribuyan con efectividad en potenciar al ambiente. Asimismo, la institución no desarrolla estrategias ambientales que de manera transversal e interdisciplinaria permitan la vinculación de las actividades y contenidos de la educación ambiental para entender $y$ atender el entorno social.

De igual manera, los profesionales de la docencia no motivan el desarrollo de habilidades ambientales que propicien las capacidades y destrezas para la aprehensión del conocimiento ambiental por parte de la comunidad estudiantil. Sin embargo, el docente tiene una actitud positiva hacía la incorporación del ecoturismo dentro del proceso educativo. De esta forma, la práctica socioeducativa que enlaza a la educación ambiental con el turismo, generará un impacto formativo caracterizado por el interés en la naturaleza; fortaleciendo el ámbito económico y social.

De acuerdo a los datos e información generada por los encuestados se 
considera la existencia de una percepción ambiental favorable y receptiva para la incorporación de estrategias ambientales en la formación ecoturística desde el escenario educativo. Por lo tanto, la vinculación de las estrategias ambientales y la formación ecoturísticas, propicia la paráfrasis y los espacios de los contextos ambientales, de las particularidades y la relevancia del patrimonio paisajístico, de las cualidades y comportamiento del individuo, de los principios ambientales contribuyendo, fundamentalmente, con la interpretación de los valores locales, regionales y universales.

En relación la factibilidad de la propuesta de estrategias ambientales en la formación ecoturística de los docentes de la Escuela Básica "Sebastián Araujo", se precisó que: la propuesta está en función del actual paradigma ambiental, relacionando los valores, en sus diferentes dimensiones, individuales y colectivos, centrado en las exigencias de atención al estudiante en el área de ambiente y turismo. El diseño de estrategias ambientales se centró en las siguientes dimensiones:

Dimensión 1. Sensibilización: Estímulo a la actitud y creatividad de cambio, la misma representa la primera actividad en la concepción de la estrategia ambiental, la cual deber poseer cada motivador ecoturístico; persiguiendo determinar la problemática social, económica, ambiental y educativa, precisando las fortalezas, debilidades, oportunidades y amenazas en el desarrollo del ecoturismo. Las estrategias ambientales que se proponen: (a) ¿Vivenciando Mis Riesgos y Acciones hacia el Ambiente?, propósito: Conocer el espacio geográfico y tiempo histórico y significativo.

Dimensión 2. Interpretación ambiental: El ecoturismo amerita de acciones educativas, en la cual se planifiquen el análisis, interpretación y reflexión sobre la temática. Por lo tanto, se focaliza la estrategia de interpretación ambiental que brindará actividades inherentes a la función educativa, social, cultural y económica del ecoturismo. Las estrategias 
ambientales que se plantean: (a) Socio Turismo-Ambiental, propósito: Conocer la importancia del ambiente en su componente natural del entorno, indagar el espacio geográfico y tiempo histórico significativo.

Dimensión 3. Formación: Dicha dimensión se centra en la formación de guías ambientales: Esta actividad tiene como propósito potenciar la generación de actitudes proactivas en espacios que concedan el discernimiento y la deliberación de temáticas relacionadas con la ecología, el turismo y el ambiente. Además, promueve los recorridos por el contexto, los espacios escolares, comunitarios y la imaginación por otros contextos exteriores. Persigue propiciar la construcción de aprendizajes significativos relacionados con el ecoturismo. La estrategia ambiental que se propone es ¿Quién soy en mi comunidad?, la misma tiene como intención, conocer el turismo y el turista como receptor del ambiente.

Por consiguiente, con el diseño de las estrategias ambientales contribuirá con la interpretación de los conceptos ambientales con pertinencia y congruencia, se podrá enfatizar entre las acciones de vida del hombre y su entorno. Consecuentemente, se requiere de un nuevo enfoque constructivista que le permita al estudiante ser promotor social y cohesione los integrantes de la comunidad educativa el mejoramiento del ambiente y e turismo a través de estrategias significativas de aprendizaje que contribuyan a consolidar el trabajo comunitario dentro y fuera de la comunidad.

En atención al análisis realizado se presentan las siguientes recomendaciones: Incorporar la propuesta buscando dar coherencia a los enfoques bajo los cuales puede ser abordada, estableciendo estrategias primarias de ejecución, tales como: (a) El análisis de la misión y la visión institucional; (b) La identificación del papel asignado al docente, alumno, y sociedad dentro del proyecto institucional; (c) Revisión de las estrategias y acciones detalladas en torno a la enseñanza; y (d) Impulsar la capacitación 
científica y técnica en materia de educación ambiental y turismo.

\section{Referencias}

Fuentes, F. (2013). Formación, Proceso Social y Cultural. Tercera edición. Madrid, España: Editorial Limusa.

Martín, T. (2010a,b). Los Principios del Ecoturismo Moderno. Caracas, Venezuela: Editorial Planeta Venezolana, S.A.

Martínez, R. (2010). La Importancia de la Educación Ambiental Ante la Problemática Actual. Revista Electrónica Educare, XIV(1), 97-111, EISSN: 1409-4258. Recuperado de:

https://www.redalyc.org/pdf/1941/194114419010.pdf

Molano, A. (2013a,b,c). Concepciones y Prácticas sobre Educación Ambiental de los Docentes en las Universidades de Bogotá. Implicaciones para los Currículos de las Facultades de Educación.

Tesis Doctoral. Valladolid, España: Universidad de Valladolid. Recuperado de:

https://uvadoc.uva.es/bitstream/10324/4238/1/TESIS447-140205.pdf

Palella, S., \& Martins, F. (2012). Metodología de la Investigación. Segunda Edición. Caracas, Venezuela: Fondo Editorial de la Universidad Pedagógica Experimental Libertador - FEDUPEL.

Rengifo, B., Quitiaquez, L., \& Mora, F. (2012). La Educación Ambiental una Estrategia Pedagógica que Contribuye a la Solución de la Problemática Ambiental en Colombia. XII Coloquio Internacional de Geocrítica. Bogotá, Colombia: Universidad Nacional de Colombia. Recuperado de: http://www.ub.edu/geocrit/coloquio2012/actas/06-BRengifo.pdf

Requena, Y. (2018). Investigación Acción Participativa y Educación Ambiental. Revista Scientific, 3(7), 289-308. Recuperado de: https://doi.org/10.29394/Scientific.issn.2542-2987.2018.3.7.15.289-308 
Romero, O. (2010a,b). Estrategias Ambientales: Una Nueva Educación Hacia el Ambiente. Segunda Edición. Madrid, España: Editorial Pita. Sánchez, J. (2012a,b). El Desafío Ambiental. Ecología y Medio Ambiente. Segunda edición. Bogotá, Colombia: Editorial Norma.

Santander, E. (2010a,b,c). Escuela y Comunidad. Caracas, Venezuela: Coordinación de Extensión de la Universidad Nacional Abierta - UNA. Universidad Pedagógica Experimental Libertador (2016). Manual de Trabajos de Grado de Especialización y Maestría y Tesis Doctorales. 5ta Ed. Caracas, Venezuela: Fondo Editorial de la Universidad Pedagógica Experimental Libertador - FEDUPEL.

Villegas, M. (2012). Conceptualización del Ecoturismo. México: Editorial Limusa.

Zuccarro, G. (2010). Educación Ambiental y Turismo Sostenible: Influencia del Ambiente en la Sensibilización y Comportamiento Medio Ambiental. Propuesta de un programa educativo ambiental para los balnearios del Estado Barinas. Tesis Doctoral. Granada, España: Universidad de Granada. Recuperado de: https://hera.ugr.es/tesisugr/2044879x.pdf 


\section{Belkis Esteban Moncada \\ e-mail: belkisesteban2016@gmail.com}

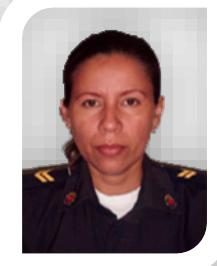

Nacida en el municipio Antonio José de Sucre, Socopó del estado Barinas, Venezuela, el 26 de septiembre del año 1978. Realicé estudios en la Unidad Educativa José Rafael Pulido Meza en el estado Barias, obteniendo el título de Bachiller en Ciencias; con estudios superiores en la Universidad Nacional Experimental de los Llanos Occidentales Ezequiel Zamora, obteniendo el título de Licenciada en Educación Integral; actualmente efectué estudios de postgrado en la Universidad Pedagógica Experimental Libertador, Instituto de Mejoramiento Profesional del Magisterio, Núcleo Académico Barinas, obteniendo el título de Maestría en Educación, Ambiente y Desarrollo. He realizado cursos tales como el 1er Congreso Internacional de Investigación e innovación en Currículo y Formación Docente, Universidad Nacional Experimental de los Llanos Occidentales Ezequiel Zamora, en noviembre del año 2015. Cuento con Experiencia laboral en Apoyo a la Gran Misión Abastecimiento Soberano, desde el mes de abril del año 2016 hasta la actualidad.

El contenido de este manuscrito se difunde bajo una Licencia de Creative Commons ReconocimientoNoComercial-Compartirlgual 4.0 Internacional 